\section{An Increase in the Prevalence of KPC Nosocomial Bacteremia as a Trigger for Growing Polymyxin Resistance Among Other Multidrug-Resistant Non-KPC-Producing Enterobacteriaceae Isolates}

To the Editor-The worldwide emergence of carbapenemases has challenged antimicrobial therapy against the Enterobacteriaceae family, which are dangerous pathogens in most nosocomial infections. ${ }^{1}$ A recent survey demonstrated that the production of this type of enzyme could be strongly related to an increased prevalence of an agent carrying it, with unfavorable clinical outcomes. ${ }^{2,3}$

Bacterial bloodstream infections (BSIs) are serious infections associated with significant mortality and healthcare costs. Although a cause-and-effect association has not yet been properly elucidated, as previously reported by Tumbarello et $\mathrm{al}^{4}{ }^{4}$ the emergence of Klebsiella pneumoniae carbapenemase (KPC)-producing Enterobacteriaceae as a BSI agent has been associated with a higher mortality rate than infections not due to a KPC-producing pathogen. Klebsiella pneumoniae carbapenemase-producing Enterobacteriaceae is the most prevalent carbapenemase in Brazil, and the prevalence of KPC has been increasing in most Brazilian hospitals, severely limiting the therapeutic options for the treatment of patients because $\beta$-lactams, including carbapenem, become ineffective, with only a few agents remaining active, particularly polymyxins. ${ }^{5}$ The current study was performed (1) to determine the cumulative prevalence of BSIs caused by KPC-producing Enterobacteriaceae, by no carbapenemase-producing multidrug-resistant (nCP-MDR) organisms, and by K. pneumoniae species with no resistance mechanism detected, (2) to measure the impact of the presence of the $b l a_{\mathrm{KPC}}$ gene, and (3) to assess the polymyxin resistance rate among these pathogens in comparison to the prevalence rate of those organisms naturally resistant to this drug.

Patients followed in a surveillance cohort performed at a general-care hospital in Porto Alegre, southern Brazil, who presented positive blood cultures with enterobacterial isolates, were included in the study. Microbiological data such as bacterial identification, antimicrobial susceptibility testing, and characterization of any resistance mechanism are described in detail elsewhere. ${ }^{6}$ The study period was from January 1 to December 26, 2016.

A total of 535 enterobacterial isolates were recovered from BSIs; of these, $152(28.4 \%)$ had the $b l a_{\text {KPC }}$ gene (notably the sole carbapenemase detected during this survey). Klebsiella pneumoniae was the only specie characterized as KPC (ie, KPC-Kp). According to the phenotypic testing, 14 isolates were ESBL producers, including $11 \mathrm{~K}$. pneumoniae, 2 Escherichia coli, and 1 Enterobacter aerogenes. Another 7 isolates (6 Enterobacter cloacae and 1 E. aerogenes) were

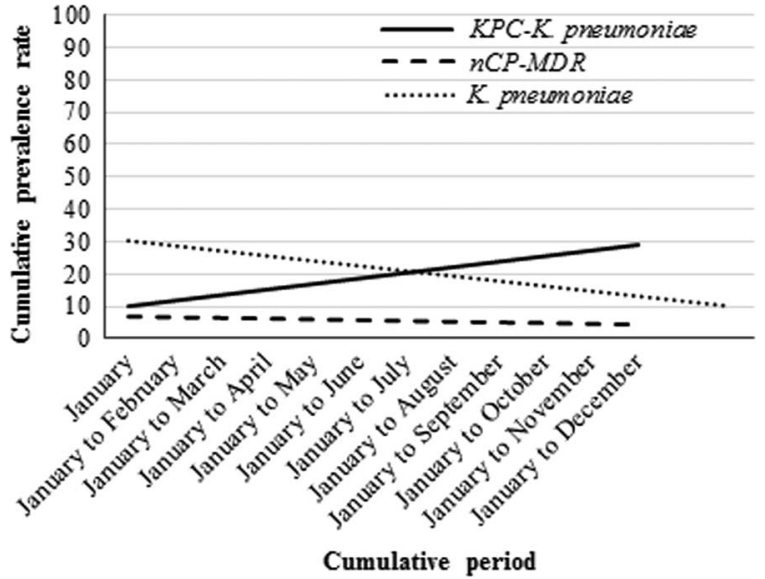

FIGURE 1. Cumulative prevalence rates of KPC, nCP-MDR, and K. pneumoniae isolates during the study period.

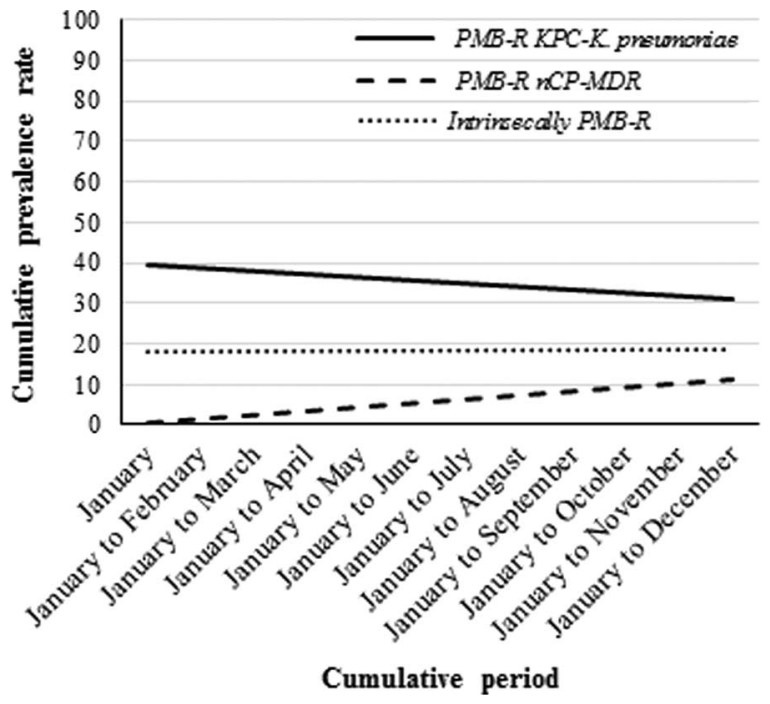

FIGURE 2. Cumulative prevalence of polymyxin-resistance rates of $\mathrm{KPC}$, nCP-MDR, and intrinsically polymyxin-resistant Enterobacteriaceae isolates during the study period.

categorized as CRE isolates because they could hydrolyze at least 1 carbapenem agent (basically ertapenem). Together, both groups comprised 21 of the isolates characterized $(3.9 \%)$ in this survey as an $\mathrm{nCP}-\mathrm{MDR}$ group. The cumulative prevalence of these enterobacterial isolates over the study period is shown in Figure 1.

An increased prevalence (media \pm standard deviation; $19.6 \% \pm 6.8$ ) was observed for KPC-Kp in BSIs, and notably, a high rate of polymyxin $\mathrm{B}(\mathrm{PMB})$ resistance $(35.3 \% \pm 11.3)$ was also observed, although a slight decreased in the trend over the assessed period was identified (Figure 2).

Although a stable cumulative prevalence $(18.5 \% \pm 1.4)$ had been observed among those organisms intrinsically resistant to PMB (an important issue in a setting with a high usage of this 
agent), we identified a trend toward increasing prevalence of polymyxin-resistant $\mathrm{nCP}-\mathrm{MDR}$ isolates $(6.1 \% \pm 4.0)$, which made the selective pressure evident, even with a reduction in the overall prevalence $(5.6 \% \pm 1.3)$ of these nCP-MDR organisms during the same study period (Figure 2).

Carbapenem-resistant $K$. pneumoniae BSIs have been associated with higher morbidity and mortality rates than BSIs due to carbapenem-susceptible isolates. ${ }^{7}$ This finding may be due to the presence of any carbapenemase gene, especially $b l a_{\mathrm{KPC}}$, which is highly endemic in Brazilian hospitals, and to the virulence and competitive fitness of $K$. pneumoniae regardless of its susceptibility profile. ${ }^{2}$

In this survey, KPC production by K. pneumoniae seems to be responsible for increasing the prevalence rate of this pathogen over the study period, compared with the prevalence rates of "wild-type" K. pneumoniae and multidrug-resistant organisms with any other mechanism (eg, ESBL, ampC, or efflux pumps). Importantly, this superior prevalence seems to be driven by the acquisition of adaptive PMB resistance, which is found mainly in KPC-Kp but also in the nCPMDR group.

Although the impact of the PMB-resistant KPC-Kp recovery on patient outcome was not evaluated in this study, the results reported here are important, particularly concerning multidrug-resistant pathogens, because KPC producers are important in the effort to reduce rates of infection, especially in a clinical site as notable as the bloodstream.

In conclusion, an increase in the prevalence of KPC-Kp recovered from the bloodstream was observed during the study period. Apart from that, KPC production probably contributes to this increased rate. A notorious emergence of polymyxin resistance among nCP-MDR isolates is worrying and may be attributed to strong selective pressure. The optimization of polymyxin use in treating BSIs must be further investigated to minimize the overall resistance development.

\section{ACKNOWLEDGMENTS}

Financial support: No financial support was provided relevant to this article. Potential conflicts of interest: The author reports no conflicts of interest relevant to this article.

\section{Leandro Reus Rodrigues Perez, PhD}

Affiliations: Hospital Mãe de Deus, Porto Alegre, Brazil

Address correspondence to Leandro Reus Rodrigues Perez, PhD, Microbiology Unit, Hospital Mãe de Deus, 286, José de Alencar Street, 90610-000, Porto Alegre RS, Brazil (leandro.reus@gmail.com).

Infect Control Hosp Epidemiol 2018;39:242-243

(C) 2018 by The Society for Healthcare Epidemiology of America. All rights reserved. 0899-823X/2018/3902-0023. DOI: 10.1017/ice.2017.269

\section{REFERENCES}

1. Tamma PD, Goodman KE, Harris AD, et al. Comparing the outcomes of patients with carbapenemase-producing and non-carbapenemase-producing carbapenem-resistant Enterobacteriaceae bacteremia. Clin Infect Dis 2016;64:257-264.

2. Rodrigues Perez LR. Carbapenem-resistant Enterobacteriaceae: a major prevalence difference due to the high performance of carbapenemase producers when compared to the nonproducers. Infect Control Hosp Epidemiol 2015;36:1480-1482.

3. Giacobbe DR, Del Bono V, Trecarichi EM, et al. Risk factors for bloodstream infections due to colistin-resistant KPCproducing Klebsiella pneumoniae: results from a multicenter case-control-control study. Clin Microbiol Infect 2015;21: 1106.e1-1106.e8.

4. Tumbarello M, Trecarichi EM, De Rosa FG, et al. Infections caused by KPC-producing Klebsiella pneumoniae: differences in therapy and mortality in a multicentric study. J Antimicrob Chemother 2015;70:2133-2143.

5. Perez LR. Know thy self, know thy enemy: a current survey and a forecast for KPC-producing Klebsiella pneumoniae resistance among inpatients in southern Brazil. Infect Control Hosp Epidemiol 2017;38:754-755.

6. Perez LR. Epidemiology of adaptive and intrinsic polymyxin resistance mechanisms by comparing polymyxin-resistant pathogens prevalence in a one-year follow-up survey. Infect Control Hosp Epidemiol 2017;38:1121-1123.

7. Tumbarello M, Viale P, Viscoli C, et al. Predictors of mortality in bloodstream infections caused by Klebsiella pneumoniae carbapenemase-producing Klebsiella pneumoniae: importance of combination therapy. Clin Infect Dis 2012;55:943-950.

\section{Validation and Evaluation of Antimicrobial Orders Indication for Use}

To the Editor-Tracking and monitoring antimicrobial prescribing, which includes documentation of the indication for use, is 1 of the 7 Core Elements of Antimicrobial Stewardship Programs set forth by the Centers for Disease Control and Prevention (CDC). ${ }^{1}$ Requiring a question for indication in the computerized physician order entry (CPOE) allows for increased transparency and communication among patients and all members of the healthcare team; it also enhances mindful prescribing, medication safety, and understanding of antimicrobial use. ${ }^{2}$ Thus, on September 20, 2016, a mandatory selection of antimicrobial indication as an empiric or pathogendirected therapy or as a prophylaxis was implemented for all prescribers within the Cleveland Clinic Health System (CCHS), which comprises an academic medical center, 8 community hospitals in Northeast Ohio, and 1 community hospital in Florida. Given the role of antimicrobial indication as a measurement of prescribing patterns and a guide for future stewardship activities, validation is important and necessary to ensure accurate selection of indications by the end users of the electronic medical record (EMR). We sought to describe the accuracy of prescriber-entered antimicrobial 